\section{Low-frequency ultrasound causes brain edema and intracerebral necrosis in rats}

The effectiveness of thrombolysis in acute stroke treatment can be enhanced by transcranial ultrasound, which has been shown to improve recanalization rates. Ultrasound in the low-frequency $(\mathrm{kHz})$ range may be more effective than high-frequency ultrasound, because low-frequency sound penetrates bone and tissue more effectively. Concerns have, however, been raised about the safety of low-frequency transcranial ultrasound; insonation at $300 \mathrm{kHz}$ was associated with increased rates of cerebral hemorrhage in a recent trial.

Researchers in Germany have assessed the safety of $20 \mathrm{kHz}$ transcranial ultrasound in rats, at power outputs ranging from $0 \mathrm{~W} / \mathrm{cm}^{2}$ to $2.6 \mathrm{~W} / \mathrm{cm}^{2}$. Treatment at an output $\geq 0.5 \mathrm{~W} / \mathrm{cm}^{2}$ was associated with cytotoxic and vasogenic edema, as measured by MRI. Ultrasound at $2.6 \mathrm{~W} / \mathrm{cm}^{2}$ caused intracerebral necrosis and loss of neurons. Tympanic temperature measurements and the histological necrosis pattern suggest that the damage was not caused by thermal effects, although the possibility of such effects cannot be completely excluded. No damage was detected after treatment at an output of $0.2 \mathrm{~W} / \mathrm{cm}^{2}$, an intensity that has been shown to be effective in in vitro thrombolysis experiments.

The study shows that low-frequency ultrasound can be harmful to healthy brain tissue, and the authors caution that damaged tissue might be more vulnerable to adverse effects of treatment. Neurological damage cased by the treatment was dose-dependent, however, and the authors state that low-intensity treatment $\left(0.2 \mathrm{~W} / \mathrm{cm}^{2}\right)$ should be further evaluated in animal models.

Original article Schneider F et al. (2006) Brain edema and intracerebral necrosis caused by transcranial low-frequency 20-kHz ultrasound: a safety study in rats. Stroke 37: 1301-1306

\section{Does long-term antihypertensive treatment affect risk of dementia?}

Studies have indicated that midlife hypertension might increase the risk of late-life cognitive impairment. Although some studies have reported that antihypertensive treatment reduces dementia risk in elderly patients, there is controversy over the long-term effects. Data from the prospective, population-based Honolulu-Heart Program/ Honolulu-Asia Aging Study, which started in 1965, have been used to investigate the risk of dementia and cognitive decline associated with various durations of antihypertensive treatment.

The analysis was based on 1,294 elderly men (born from 1900 to 1919) who had been hypertensive since middle age; 108 of these patients were diagnosed with dementia during follow-up. Treated hypertensives $(n=848)$ had received an average of 12.8 years of antihypertensive treatment and, compared with nontreated hypertensives, the risk of dementia was reduced by about $5 \%$ for each year of antihypertensive treatment. Hypertensive men who had received $\leq 5$ years of antihypertensive treatment, however, were at similar risk of dementia to hypertensives who had never received treatment. Treatment duration affected the risk of dementia, with hypertensive patients who had been treated for $\geq 12$ years showing a similar risk of dementia to normotensives; this 'treatment duration effect' occurred mainly among patients with controlled blood pressure. Cognitive function declined with age in patients without dementia; hypertensive patients without dementia who had been treated with antihypertensives for 5-12 years, however, showed a lower rate of decline than hypertensives who had never received treatment.

The authors recommend that the finding that increased duration of antihypertensive treatment reduces the risk of dementia and cognitive decline is tested in further trials.

Original article Peila R et al. (2006) Reducing the risk of dementia: efficacy of long-term treatment of hypertension. Stroke 37: 1165-1170

\section{Epstein-Barr virus levels elevated up to 20 years before onset of multiple sclerosis}

In a recent prospective study, researchers investigated levels of antibodies to the Epstein-Barr virus (EBV) in patients with multiple sclerosis (MS), using blood samples taken several decades before they developed disease symptoms. Increased EBV antibody levels have previously been reported in patients with MS up to 10 years before symptom onset.

DeLorenze and colleagues identified 42 patients with MS who were members of the Kaiser Permanente Northern California health 